\title{
AUTOEVALUACIÓN, PLAN DE MEJORAS Y EFICACIA ACADÉMICA EN LA FACULTAD DE CIENCIAS ADMINISTRATIVAS DE LA UNIVERSIDAD NACIONAL MAYOR DE SAN MARCOS
}

\author{
SELF-ASSESSMENT, IMPROVEMENT PLAN AND EFFECTIVE ACADEMIC IN THE FACULTY OF \\ ADMINISTRATIVE SCIENCES OF THE BIGGEST NATIONAL UNIVERSITY OF SAN MARCOS \\ Edwin Augusto Vigo Sánchez* \\ evigos@unmsm.edu.pe
}

\begin{abstract}
RESUMEN
La presente investigación tiene el propósito de contribuir al logro de la acreditación de la Facultad de Ciencias Administrativas de la UNMSM, en el marco de la Ley del SINEACE No. 28740, Ley del Sistema Nacional de Evaluacion de la Calidad Educativa, encargada de normar los procesos de evaluacion, acreditación y certificación de la calidad educativa en nuestro país.

Un proceso de autoevaluación debe conducir a una posterior acreditación y certificación de la facultad, ello implica el desarrollo, análisis y retroalimentación relacionados a las prácticas preprofesionales, competencias adquiridas por estudiantes y graduados, metodología para la evaluación y seguimiento del aprendizaje; organización administrativa, servicios académicos complementarios, estructura física y equipamiento.

La autoevaluación, es un proceso de análisis crítico de cada una de las unidades académicas y áreas administrativas de la facultad, que precede a la acreditación.

La acreditación es un procedimiento, cuyo objetivo es comparar el grado de acercamiento del objeto analizado con un conjunto de normas previamente definidas e implantadas como deseables.

El objetivo de la Facultad de Ciencias Administrativas de la Universidad Nacional Mayor de San Marcos, consiste en formar profesionales competentes y de alto nivel académico, comprometidos con el desarrollo del país mediante la investigación científica y humanista y la conservación del medioambiente.

Palabras claves: Evaluación, acreditación, certificación, retroalimentación, aprendizaje, medio ambiente.
\end{abstract}

\footnotetext{
* Doctor en Administració, Docente permanente de la Facultad de Cincias Administrativas de la Universidad Nacional Mayor de San Marcos, asesor y consultor empresarial en el área de Recursos Humanos, docente de posgrado de diversas universidades públicas y privadas.
} 


\begin{abstract}
This research has the purpose of contributing to the achievement of the accreditation of the school of Sciences administrative from San Marcos University, within the framework of the law of the SINEACE No. 28740 , law on the national system of evaluation of the quality of education, responsible for standardizing the processes of evaluation, accreditation and certification of the quality of education in our country.

a process of self-assessment should lead to a subsequent accreditation and certification by the faculty, entails the development, analysis and feedback related to the practices pre professionals, skills acquired by students and graduates, methodology for the evaluation and monitoring of learning; administrative organization, complementary academic services, structure physics and equipment.

The self-assessment is a process of analysis critical of academic and faculty administrative areas, units that precedes the accreditation.

Accreditation is a procedure, whose objective is to compare the degree of approach of the object analyzed with a set of rules previously defined and implemented as desirable.

The Faculty of sciences administrative of the University National chief of San Marcos aims to train competent professionals and high level academic, committed to the development of the country through the research scientist and humanist and the preservation of the environmental conservation.
\end{abstract}

Keywords: Assessment, accreditation, certification, feedback, learning, environment.

\section{INTRODUCCIÓN}

Para insertarse con efectividad al mundo globalizado, en la era del conocimiento, las universidades actualmente no solo requieren modernizarce y perfeccionar sus mecanismos democraticos, sino, también, una mejora continua de la calidad de la enseñanza y de la investigación multidisciplinaria, con las que se podra aspirar a la excelencia académica e institucional, que serán garantía pública de ser un espacio de producción de conocimiento para la formación de cuadros altamente calificados.

Respecto a la calidad, Unesco (1995) señala, que la calidad ha llegado a ser una de las preocupaciones fundamentales de la educación superior ello obedece a que la satisfacción de las expectativas y las necesidades de la sociedad en relación con la educación superior depende, en ultima instancia de la calidad de los recursos humanos, sus programas y estudiantes, así como de su estructura y su entorno académico. la busqueda de la calidad tiene multiples facetas y el principal objetivo de las medidas tendientes a aumentar esa calidad deberá ser el automejoramiento tanto de sus instituciones como de todo el sistema.

Las universidades están constituidas por un conjunto de facultades que albergan docentes, estudiantes y personal administrativo. Las facultades de Ciencias Administrativas son una de las facultades que existen en casi el $100 \%$ de las universidades públicas y privadas del perú, específicamente en la Universidad Nacional Mayor de San Marcos.
Las iniciativas para lograr la calidad se vienen dando en diversas universidades e instituciones educativas de nivel superior tanto nacionales como internacionales. Así tenemos que los autores Valencia R., Hernandez \& Gonzales R.(2003), realizaron una investigación titulada "Hacia un modelo de autoevaluación universitaria", la cual tuvo como objetivos: desarrollar un conjunto de parámetros precisos, válidos y aplicables a la situación real de la institución que permitan evaluar el ser y el quehacer del servicio educativo; elaborar un modelo de autoevaluación que nos ayude de manera rápida y eficiente a detectar las condiciones, las fortalezas y debilidades de manera veraz, rapida y consistente y, la presentación de un documento que sintetice los resultados de la aplicación del modelo, resaltando las áreas que requieran atención.

La UNMSM ha sido la primera universidad publica que inició su autoevaluación, en el 2003, desde entonces se han dado propuestas concretas como las de Orellana, O. \& Orellana, D. (2004), en relación a la matriz de autoevaluación universitaria para la carrera de Psicología que constituye la segunda parte de una investigación exploratoria. En esta propuesta de una matriz de autoevaluación universitaria de la Facultad de Psicología de la UNMSM, se precisa el carácter de la autoevaluación, los objetivos de mejora y la acreditación. asi como las fases que transita el proceso de autoevaluación, concluyendo con la matriz compuesta por veinte factores, haciendo una propuesta de indicadores y fuentes de verificación. 


\section{PLANTEAMIENTO DEL PROBLEMA}

\section{EL problema}

\section{El problema general}

¿En qué medida la autoevaluación y el establecimiento de un plan de mejoras influirán en la eficacia académica de la Facultad de Ciencias Administrativas de la UNMSM?

\section{Problemas específicos}

a) ¿En qué medida la autoevaluación influirá en la eficacia académica de la Facultad de Ciencias Administrativas de la UNMSM?

b) ¿En qué medida el establecimiento de un plan de mejoras influirá en la eficacia académica de la Facultad de Ciencias Administrativas de la UNMSM?

\section{OBJETIVOS}

Determinar la influencia de la autoevaluación en el logro de la eficacia académica de la Facultad de Ciencias Administrativas de la UNMSM.

Determinar la influencia del establecimiento de un plan de mejoras, en el logro de la eficacia académica de la Facultad de Ciencias Administrativas de la UNMSM.

\section{JUSTIFICACIÓN}

La presente investigación se justifica por la inserción del país en la aldea global, lo cual implica que sus organizaciones, y en especial las universidades, desarrollen una cultura de cambio reflejada en una nueva visión del mundo, nuevos métodos pedagógicos, nuevos enfoques de aprendizajes, nuevas tecnologías educativas, nuevos sistemas de información, nuevos niveles de competitividad y nuevas carreras técnicas y profesionales. Estos nuevos cambios suponen adecuarse a estándares internacionales de calidad.

Es importante, además, porque permitirá plantear propuestas de cambio en la Facultad de Ciencias Administrativas de la UNMSM, la cual tiene la responsabilidad de formar profesionales líderes para desempeñarse en las micro, pequeñas, medianas y grandes empresas de nuestro país, de allí la importancia de abordar el tema de la autoevaluación, plan de mejoras y eficacia académica en la Facultad de Ciencias Administrativas de la UNMSM.

\section{HIPÓTESIS}

La autoevaluación y el establecimiento de un plan de mejoras, contribuirán al logro de la eficacia académica en la Facultad de Ciencias Administrativas de la Universidad Nacional Mayor de San Marcos.

\section{METAS ESPECÍFICAS}

Lograr que el $80 \%$ de los docentes nombrados se involucren en el desarrollo de proyectos de investigacion.

Lograr que el $100 \%$ de los docentes nombrados tengan el grado de maestro en su especialidad y el $50 \%$ de los mismos tengan el grado de doctor.

Implementar nuevos planes curriculares, acorde con las exigencias del mercado laboral.

\section{MARCO TEÓRICO DEL TRABAJO}

La acreditación universitaria es el resultado de un proceso de evaluación y seguimiento sistemático y voluntario del cumplimiento de las funciones universitarias de una institución de educación superior (IES), que permite obtener información fidedigna y objetiva sobre la calidad de las instituciones y programas universitarios que desarrolla. Permite certificar, ante la sociedad, la calidad de los recursos humanos formados y de los diferentes procesos que tienen lugar en una institución educativa. 1 Es el reconocimiento formal y público otorgado a una institución académica en virtud del grado en que dicha institución, o dentro de esta, una o más de sus unidades, carreras o programas han logrado avances significativos en el cumplimiento de su misión y objetivos declarados, y satisface un conjunto acordado de criterios, indicadores y estándares de pertinencia y calidad. Descansa en la búsqueda permanente de la excelencia y representa el esfuerzo colectivo de la comunidad universitaria para rendir cuentas a sí misma y a la sociedad, sobre la pertinencia, relevancia y calidad de su ser y quehacer institucional.2

En la medida en que crecen y se diversifican las actividades y ofertas educacionales, investigativas y de extensión de una institución universitaria, se hace indispensable que existan y operen efectivamente mecanismos de control, cohesión y confiabilidad pública respecto de ellos. Esta necesidad tiene su máxima expresión en el sistema de acreditación. 


\section{Propósito, características y dimensiones de la acreditación universitaria}

El propósito central de la acreditación es promover y estimular el continuo mejoramiento y determinar si una institución académica posee calidad a nivel general o respecto de una o más de sus carreras o programas educativos; si es capaz de demostrar que progresa de manera continua y sistemática, con el empleo de estrategias, procedimientos y recursos adecuados para el logro de su misión y sus objetivos, cumpliendo razonablemente con los criterios y normas de calidad establecidos.

La acreditación no es permanente, sino que se otorga por un periodo que puede variar entre 5 y 10 años, a partir del cual puede ser renovada o retirada, sobre la base de los resultados obtenidos en el proceso de revisión o verificación efectuado.

Consecuentemente, la acreditación universitaria se concibe como un mecanismo mediante el cual la comunidad educativa establece y mantiene su autorregulación, y garantiza a los usuarios directos e indirectos de los servicios que ofrece, su integridad, pertinencia y calidad, en niveles que la hagan merecedora de la confianza y el respeto público.

La gran meta de la universidad latinoamericana es responder a una triple misión:3

- Hacer socialmente relevante su capacidad de creación cultural, de innovación científica y tecnológica y de formación de recursos humanos.

- Extender, por equidad, los conocimientos generados y acumulados en la institución a sectores amplios de la sociedad.

- Asegurar la excelencia por el mantenimiento y la mejora de la calidad de la enseñanza, investigación y extensión.

La evaluación institucional es, primordialmente, la valoración del nivel de calidad o excelencia de un centro educativo; pero, a la vez, la calidad es, además de objeto de la evaluación, meta, fin y hasta criterio de la propia evaluación; es decir, la calidad es el modelo organizado de la propia evaluación.

Se consideran dos dimensiones o enfoques de la calidad de la educación: 4

- Satisfacción del educando o la familia como usuario o consumidor externo y satisfacción del trabajador (profesor, ejecutivo, administrador) como consumidor interno.
- Características del producto y su ajuste a determinados estándares predeterminados. Naturaleza de la educación, integridad de todos los factores del desarrollo humano, orden y coherencia en la relación lógica entre los factores que la componen y la eficacia del cumplimiento de sus funciones en cada uno de los procesos universitarios.

Ambas dimensiones son necesarias y complementarias; la primera insiste en el para quién (a quién satisface), y la segunda en el qué y cómo (qué es la buena educación). La calidad en la educación es el efecto resultante de una relación peculiar entre los componentes básicos, internos y externos del sistema o del centro educativo. Al respecto se consideran los estándares de calidad siguientes:5

- De tipo científico: validez, confiabilidad, generalizabilidad y objetividad.

- De tipo práctico-social: pertinencia, importancia, suficiencia, credibilidad, oportunidad, amplitud de difusión.

Es importante la evaluación tanto de los resultados como de los procesos en sí mismos.

TABLA 1. Fuente de necesidades de la evaluación institucional

(Tomado de Cañete Escalona F.)5

\begin{tabular}{|c|c|c|}
\hline No. & Fuente & Situación \\
\hline 1 & $\begin{array}{l}\text { Apreciación } \\
\text { empírica de } \\
\text { la rutina }\end{array}$ & $\begin{array}{l}\text { - En dirección de programas de } \\
\text { trabajo. } \\
\text { - En el desarrollo del currículo. } \\
\text { - En el desempeño del personal } \\
\text { docente. }\end{array}$ \\
\hline 2 & $\begin{array}{l}\text { Indicación } \\
\text { científica } \\
\text { que exige } \\
\text { toma de } \\
\text { decisiones } \\
\text { para un } \\
\text { cambio. }\end{array}$ & $\begin{array}{l}\text { - Necesidad de desarrollo de la } \\
\text { calidad institucional. } \\
\text { - Calidad rendimiento de los edu- } \\
\text { candos. } \\
\text { - Producción intelectual y científica } \\
\text { del personal docente. } \\
\text { - Intervención Universidad/Sociedad// } \\
\text { Organización. }\end{array}$ \\
\hline 3 & $\begin{array}{l}\text { Apreciación } \\
\text { científica } \\
\text { derivada } \\
\text { de inves- } \\
\text { tigaciones } \\
\text { previas. }\end{array}$ & $\begin{array}{l}\text { - En la tecnología utilizada. } \\
\text { - En la gestión institucional. } \\
\text { - En la utilización de los recursos } \\
\text { disponibles. } \\
\text { - En la interacción escuela-comun- } \\
\text { idad. }\end{array}$ \\
\hline
\end{tabular}


La Universidad ha sido creada para la excelencia en razón de su quehacer relacionado con: 6

- El conocimiento, la ciencia, la cultura, la investigación.

- El capital humano, profesionales y especialistas de las distintas ramas del saber que prepara.

- Los valores que reproduce y construye.

- El impacto en el tipo de desarrollo económico y social con el que está comprometida e impulsa.

- La independencia y responsabilidad de poder pronunciarse sobre los problemas éticos y sociales (que la sociedad necesita para reflexionar).

- La comprensión y actuación en el valor agregado de sus egresados y profesores.

El término excelencia tiene dos grandes acepciones: 7

- Sinónimo de calidad. Es el mejoramiento y renovación sistemática y constante. Destacarse pero a partir de una base de comparación relativa.

- Procesos diferentes. Es la más válida; se considera la calidad como el requisito mínimo, pues no se puede aceptar que una IES funcione sin el mínimo de calidad, y alcanzado ese mínimo (dentro de una sana práctica de competitividad) tiene la posibilidad de superarse en relación con sí misma y las otras. Esa superación en ambas direcciones, después de haber conquistado un nivel mínimo de calidad, es lo que se conceptualiza como excelencia.

La excelencia es lo que excede la calidad.

Para determinar la calidad de una institución o programa se tendrá en cuenta: 8

- Las características universales expresadas en sus notas constitutivas. Estas características sirven como fundamento de la tipología de las instituciones y constituyen los denominadores comunes de cada tipo.

- Los referentes históricos; es decir, lo que la institución ha pretendido ser, lo que históricamente han sido las instituciones de su tipo y lo que en el momento histórico presente y en la sociedad concreta se reconoce como el tipo al que esta institución pertenece (las normas existentes, las orientaciones básicas que movilizan el sector educativo).

- Lo que la institución singularmente considerada define como su especificidad o su vocación primera (la misión institucional y sus propósitos).

La calidad de una institución se reconoce, en principio, mediante los elementos clave siguientes:

- Las características del capital humano de su comunidad académica en relación con el campo de acción en que opera (tipo de conocimiento que cultiva y niveles de desarrollo alcanzado y prospectivo), así como su producción científica e intelectual.

- Las disciplinas, profesiones, ocupaciones u oficios para los cuales forma.

- La calidad y variedad de las actividades de posgrado que ofrece, tanto académicas como de superación profesional.

- Los proyectos de investigación en que participa.

- Los convenios e intercambios nacionales e internacionales suscritos.

- La relación que guarda y el compromiso que asume con la sociedad a la que pertenece.

La autoevaluación institucional, la evaluación externa y la evaluación integradora final o síntesis son componentes o etapas del proceso de acreditación; expresan, para ese propósito, un modo de conferirse la distinción antes establecida y el énfasis dado en cada uno de sus elementos.

La autoevaluación consiste en el autoestudio llevado a cabo por las mismas instituciones o programas, sobre la base de los criterios, características, variables e indicadores definidos; la institución asume el liderazgo de este proceso y propicia la participación amplia de la comunidad académica en él.

La evaluación externa o por pares, que utiliza como punto de partida la autoevaluación, identifica las condiciones internas de operación, verifica los resultados de la autoevaluación y concluye en un juicio sobre la calidad del programa.

Se consideran evaluadores externos: 
- Profesores y profesionales experimentados y reconocidos en el correspondiente campo disciplinario o como directivos de los diferentes tipos de instituciones de educación superior existentes en el país o en el exterior.

- Personas que se destacan por poseer los requisitos esenciales que caracterizan "el deber ser" de los miembros de una comunidad educativa y, que por su formación, reúnen las condiciones para evaluar el proyecto académico y el modo como se concreta en la institución, así como establecer las conexiones existentes entre lo universal, lo regional y lo local.

TABLA 2.Tipos de evaluadores (Tomado de Cañete Escalona F.) 5

\begin{tabular}{|c|c|c|}
\hline Tipos & Ventajas & Desventajas \\
\hline $\begin{array}{l}\text { Evaluadores } \\
\text { internos }\end{array}$ & $\begin{array}{l}\text { Conocen mejor } \\
\text { la organización } \\
\text { en la cual ac- } \\
\text { cionan. } \\
\text { - Poseen mayor } \\
\text { experiencia di- } \\
\text { recta en el func- } \\
\text { ionamiento de la } \\
\text { institución para } \\
\text { poder percibir } \\
\text { qué anda bien y } \\
\text { qué anda mal. } \\
\text { Disposición de } \\
\text { tiempo más flex- } \\
\text { ible para evaluar. }\end{array}$ & $\begin{array}{l}\text { Es posible que } \\
\text { determinadas } \\
\text { falencias no } \\
\text { las adviertan y } \\
\text { si lo hacen, no } \\
\text { les den impor- } \\
\text { tancia. } \\
\text { Tendencia a } \\
\text { presentar el } \\
\text { lado más posi- } \\
\text { tivo posible. } \\
\text { Pueden } \\
\text { presentarse } \\
\text { situaciones de } \\
\text { evaluaciones } \\
\text { arbitrarias. }\end{array}$ \\
\hline $\begin{array}{l}\text { Evaluadores } \\
\text { externos }\end{array}$ & $\begin{array}{l}\text { El juicio puede } \\
\text { estar menos } \\
\text { comprometido } \\
\text { con ciertos } \\
\text { intereses, } \\
\text { difíciles de no } \\
\text { tomar en cuenta } \\
\text { si pertenecen a } \\
\text { la institución. } \\
\text { El aspecto for- } \\
\text { mal puede dar } \\
\text { mayor seriedad } \\
\text { al proceso. } \\
\text { La apreciación } \\
\text { puede estar me- } \\
\text { jor relacionada } \\
\text { con situaciones } \\
\text { globales. }\end{array}$ & $\begin{array}{l}\text { Tiende a ver } \\
\text { más defi- } \\
\text { ciencias que } \\
\text { aciertos. } \\
\text { Los miembros } \\
\text { de las uni- } \\
\text { dades ejecu- } \\
\text { toras ocultan } \\
\text { o disminuyen } \\
\text { deficiencias } \\
\text { reales por te- } \\
\text { mor al uso que } \\
\text { la superioridad } \\
\text { pueda darles. } \\
\text { Están lim- } \\
\text { itados en el } \\
\text { tiempo y en la } \\
\text { normatividad. }\end{array}$ \\
\hline
\end{tabular}

La evaluación integradora final o síntesis es la realizada por una comisión o consejo nacional designado por la institución rectora estatal o asociaciones nacionales o ambas, sobre la base de los resultados de la autoevaluación y de la evaluación externa. A su término se configura el reconocimiento de la calidad que servirá como base al ministro o secretario de Educación o Educación Superior del país, para expedir el documento oficial de acreditación u optar por hacer recomendaciones para el mejoramiento de la institución o del programa y su futura reevaluación.

Cada uno de los procesos o etapas poseen un valor propio, pero se complementan y refuerzan mutuamente; los tres son importantes y juntos, le otorgan, al proceso en su conjunto, el carácter de integralidad.

\section{Evaluación institucional o autoevaluación}

La evaluación institucional o autoevaluación constituye un instrumento de gran capacidad para mejorar la calidad de una IES. Permite detectar los puntos fuertes y débiles o áreas a mejorar. Es un proceso de aprendizaje continuado y una mejora constante de la universidad.9 Permite valorar:

La forma en que la institución se aproxima a las metas preestablecidas y cómo procede para el completo desarrollo de la producción y distribución del conocimiento.

- La docencia, la investigación y los servicios.

- El desarrollo en comparación con sí misma.

- La evaluación universitaria, evaluación institucional o autoevaluación corresponde a un conjunto de prácticas, métodos, conocimientos bien diversificados y progresivamente desarrollados en el transcurso de los años, con el objeto de dar respuesta a una necesidad específica: la de una mejor gestión de la universidad.

Evaluar una universidad quiere decir emitir un juicio de valor a propósito de la pertinencia y la razón de ser de sus objetivos, su grado de cumplimiento y la forma en que han sido alcanzados.10

La evaluación institucional es una actividad de investigación y análisis, realizada por los propios agentes universitarios, destinada a verificar el nivel de logros que está alcanzando la institución, sus unidades o programas, de acuerdo con su razón de ser, y determinar además los elementos que están actuando en la indicación de menor o mayor capacidad de estos logros. Esta investigación conlleva un informe auto evaluativo que es prerrequisito y documento base para la realización de la evaluación externa. 
Para los dirigentes universitarios, la evaluación institucional constituye una herramienta esencial con el fin de garantizar patrones de calidad académico-científica, indispensables para la planificación y definición de políticas estratégicas y la gestión. También permite rendir cuentas a la sociedad sobre el desempeño de la universidad en la utilización del financiamiento gubernamental y el cumplimiento de su misión pública.3

La autoevaluación es un proceso interno de planificación, identificación, análisis crítico y prospectivo sobre la evolución y desarrollo académico alcanzado por la institución en una profesión. Se busca, por lo tanto, interpretar y valorar, mediante un proceso participativo, dialogal, reflexivo y crítico, el estado de avance de una carrera universitaria en los diversos aspectos que en conjunto definan el funcionamiento y su estructura, en la consolidación, validación y comunicación del conocimiento que le es propio. Tal sistema conlleva un proceso de carácter cíclico que verifica la pertinencia de metas, estrategias, asignación de recursos y compromisos de gestión efectiva. Es organizado y conducido por sus propios integrantes, a la luz de las circunstancias de la institución: objetivos, alcances, metas y medios. 11

Como primer paso para la acreditación universitaria, la autoevaluación es un modo de concebir la acción educativa en el que la comunidad académica de la carrera revisa su misión, reorganiza sus recursos, evalúa sus resultados y se autorregula. Asimismo, se provee de información confiable que da testimonio del grado de cumplimiento de los objetivos. Contribuye, además, a desarrollar una comunidad académica más sólida que comparte compromisos institucionales y forma profesionales altamente competitivos para un mercado de trabajo que demanda una serie de atributos, tales como una excelente preparación en ciencias básicas y tecnologías básicas, lo que debe traducirse en la habilidad para desarrollar la capacidad técnica, creatividad, facilidad para interactuar interdisciplinariamente, capacidad de síntesis, alto grado de responsabilidad y conducta ética, así como también la habilidad para el manejo de los aspectos económicos de la profesión y la conciencia y compromiso social.

Por otro lado, son elementos de la calidad de una carrera, sus insumos, el proceso, los resultados, que permiten valorar el impacto que tiene en la sociedad. La calidad de una carrera se fundamenta principalmente en los procesos de enseñanza y aprendizaje, es decir, considerando a este como el núcleo o corazón de un programa bajo el supuesto de que sus resultados determinan la calidad del graduado, sus valores y los ideales del deber ser y de su desempeño profesional.

Un requisito esencial para el éxito de la autoevaluación es que los actores involucrados directamente en las actividades específicas que se valoran sean quienes lleven a cabo este proceso de análisis y reflexión.

El proceso de autoevaluación es fundamental e imprescindible para que cualquier carrera pueda acceder al proceso de acreditación, ya que aporta información, juicios, criterios y procedimientos de cumplimiento de las normas, base necesaria para satisfacer los estándares de calidad y lograr el reconocimiento público. El incremento de la calidad de las carreras es una aspiración de desarrollo tanto individual como colectivo, y es vista como un desafío, una necesidad y un compromiso con el que se responsabilizan los estudiantes, el personal docente, la carrera y la institución. 12

\section{Los objetivos de la autoevaluación son: 5,10}

- Determinar la medida del cumplimiento de los objetivos propios de la institución.

- Conocer el estado de funcionamiento de los factores de factibilidad que inciden en el logro de los objetivos.

- Indagar con más o menos precisión sobre la capacidad real y potencial de logros de la institución.

- Disponer de información confiable para regular a tiempo las estrategias de desarrollo institucional.

- Informarse del estado del desarrollo y calidad de los diferentes procesos institucionales: educacionales, investigativos y de extensión.

- Informarse del estado y funcionamiento de las instalaciones y servicios que brinda la institución.

- Informarse del estado de los recursos humanos, materiales y financieros y su uso.

- Relacionarse con el mundo exterior local, nacional e internacional.

- Identificar fortalezas y debilidades y determinar oportunidades y amenazas. 
- Proveer a las autoridades superiores en relación con la situación global de la institución, de la información que se estime necesario deba conocer.

- Comparar el mejoramiento institucional logrado en cuanto a capacidad de consecución de las metas, por periodos de tiempo, sobre la base de los recursos humanos y financieros razonablemente asignados a la institución.

- Plantear soluciones alternativas en la búsqueda de la eficiencia interna y la eficacia institucional.

TABLA 3. Cambios y problemas en la educación superior (Tomado de Kent R.) 13

\begin{tabular}{|c|c|c|}
\hline Aspecto & Cambios & Problemas \\
\hline $\begin{array}{l}\text { Institu- } \\
\text { ciones }\end{array}$ & $\begin{array}{l}\text { - Diversificación y } \\
\text { creación de nuevos } \\
\text { tipos de institucio- } \\
\text { nes. } \\
\text { Competencia entre } \\
\text { sectores. }\end{array}$ & $\begin{array}{l}\text { Crecimiento des- } \\
\text { regulado. } \\
\text { Falta de claridad } \\
\text { sobre el desem- } \\
\text { peño institucional }\end{array}$ \\
\hline $\begin{array}{l}\text { Estudi- } \\
\text { antes }\end{array}$ & $\begin{array}{l}\text { - Incorporación de } \\
\text { nuevos estratos so- } \\
\text { ciales e incremento } \\
\text { de participación } \\
\text { femenina. } \\
\text { Diversificación ti- } \\
\text { pos de estudiantes } \\
\text { (tiempo parcial, } \\
\text { estudios combina- } \\
\text { dos con trabajo, } \\
\text { etc.). }\end{array}$ & $\begin{array}{l}\text { - Alto nivel de de- } \\
\text { serción. } \\
\text { Baja eficacia ter- } \\
\text { minal. } \\
\text { - } \\
\text { Reducción de bar- } \\
\text { reras para el in- } \\
\text { greso seguido por } \\
\text { fuerte selección. } \\
\text { Falta de adecuación } \\
\text { curricular a las } \\
\text { nuevas demandas. }\end{array}$ \\
\hline $\begin{array}{c}\text { Aca- } \\
\text { démicos }\end{array}$ & $\begin{array}{l}\text { - Incorporación de } \\
\text { gran número de } \\
\text { profesores. } \\
\text { - Creación de } \\
\text { nuevas funciones } \\
\text { (tiempo completo, } \\
\text { investigador, etc.). }\end{array}$ & $\begin{array}{l}\text { - } \quad \text { Bajo nivel de } \\
\text { preparación por } \\
\text { contratación } \\
\text { apresurada. Caída } \\
\text { salarios reales en } \\
\text { el } 40 \text { a } 50 \% \text {. } \\
\text { Bajos niveles de } \\
\text { producción cientí- } \\
\text { fica. }\end{array}$ \\
\hline Gestión & $\begin{array}{l}\text { Surgimiento } \\
\text { administración } \\
\text { universitaria como } \\
\text { función específica } \\
\text { y compleja. } \\
\text { - Diversificación } \\
\text { interna de las } \\
\text { instituciones. }\end{array}$ & $\begin{array}{l}\text { Caducaron las for- } \\
\text { mas tradicionales: } \\
\text { administración de } \\
\text { aficionados, órga- } \\
\text { nos colegiados. } \\
\text { - Lento desarrollo de } \\
\text { nuevas formas de } \\
\text { gestión. } \\
\text { Burocratización } \\
\text { anárquica. } \\
\text { Baja eficiencia } \\
\text { financiera. }\end{array}$ \\
\hline
\end{tabular}

TABLA 4. Niveles del sistema y posibles definiciones (Tomado de Kent R.) 13

\begin{tabular}{|c|c|c|}
\hline Nivel & Tareas & Posibles definiciones \\
\hline $\begin{array}{l}\text { Gobi- } \\
\text { erno }\end{array}$ & $\begin{array}{l}\text { - Financiación. } \\
\text { - Acreditación. } \\
\text { - Definición de } \\
\text { prioridades. } \\
\text { - Diferenciación de } \\
\text { opciones. }\end{array}$ & $\begin{array}{l}\text { - Adecuado marco } \\
\text { regulatorio. } \\
\text { - Clara definición de } \\
\text { metas, estándares } \\
\text { e instrumentos } \\
\text { formulados en las } \\
\text { políticas. }\end{array}$ \\
\hline $\begin{array}{l}\text { Institu- } \\
\text { ciones }\end{array}$ & $\begin{array}{l}\text { - Gestión. } \\
\text { - Organización } \\
\text { académica. } \\
\text { - Política estudiantil. } \\
\text { - Atención a la } \\
\text { comunidad. }\end{array}$ & $\begin{array}{l}\text { - Definición de la } \\
\text { misión. } \\
\text { - Formulación de } \\
\text { estrategias adec- } \\
\text { uadas. } \\
\text { - Gestión requerida } \\
\text { para las estrate- } \\
\text { gias. }\end{array}$ \\
\hline $\begin{array}{l}\text { Uni- } \\
\text { dades }\end{array}$ & $\begin{array}{l}\text { - Organización } \\
\text { académica. } \\
\text { - } \quad \text { Currículo. } \\
\text { - } \\
\text { Atención a estudi- } \\
\text { antes. }\end{array}$ & $\begin{array}{l}\text { Diversas, aten- } \\
\text { diendo a la misión } \\
\text { institucional y la } \\
\text { disciplina o pro- } \\
\text { fesión. }\end{array}$ \\
\hline
\end{tabular}

Por tanto, la autoevaluación de una carrera es un proceso que conlleva cambios a corto, mediano y largo plazos y contribuye a que la institución reflexione y evalúe el grado de cumplimiento de su misión, las metas y objetivos derivados de esta, a conocer el éxito honesta y realmente, a explorar modos y medios para mejorar la eficiencia y efectividad tanto educativa como operativa, y a prepararse mejor para responder a las cambiantes y crecientes demandas de la sociedad a la que sirve.

El proceso de evaluación institucional debe estar orientado hacia un triple objetivo: 3

- Mejorar la calidad de la educación superior que desarrolla.

- Mejorar la gestión universitaria.

- Rendir cuentas a la sociedad.

\section{Aportes de la autoevaluación}

- Los aportes y beneficios de la autoevaluación son los siguientes:

- Se logra optimizar el proceso de organización y planificación de la carrera.

- Se incrementa el conocimiento de lo que la unidad académica y la carrera poseen.

- Se favorece la objetividad en la revisión de los objetivos y metas de las actividades que se realizan. 
- Se logra una sólida base de datos mejor organizadas que facilita la toma de decisiones en la gestión institucional.

- Se identifican y dimensionan las fortalezas, las debilidades y las áreas problemáticas.

- Se aumenta la comunicación en la unidad académica.

- Se facilita la asignación interna de recursos.

- Se propicia el autoanálisis reflexivo que provoca un mayor grado de identidad y de compromiso de los actores con la misión institucional.

- Se verifica la coherencia de la carrera con el perfil del ingreso, de egreso profesional, el currículo y el entorno laboral.

- Se constata si lo que se hace, se hace correctamente.

- Se programan acciones específicas derivadas de sus resultados.

- Se mejora la gestión y con esto la posibilidad de que aumente el flujo de recursos para la carrera.

Como condiciones mínimas para obtener los beneficios anteriores, deben tenerse en cuenta los siguientes factores: 11

- Motivación interna por una cultura de evaluación con incentivos propios.

- Liderazgo, planeamiento y control no fiscalizador, sino de progreso, desarrollo.

- Compromiso institucional y apertura al cambio.

- Acciones concretas sobre los resultados de la autoevaluación.

- Participación y apropiación del proceso y resultados.

- Respeto por la adecuada reserva de la información.

- Localización por áreas de los problemas importantes detectados.

- Empleo y manejo de información relevante.

- Establecimiento de ciclos periódicos de autoevaluación para la carrera.

- Provisión de una mínima capacitación interna.
- Garantía de un clima de confianza.

\section{Obstáculos principales de la evaluación institucional}

La resistencia al cambio: un sector mayoritario rechaza la evaluación universitaria en defensa de sus actuales niveles de ineficiencia y baja calidad y esgrime para ello la "autonomía universitaria", lo que se debe fundamentalmente a los siguientes elementos: 12

- No existe o no está arraigada una cultura evaluativa.

- Existe clima de desconfianza frente a un modelo de evaluación ligado al presupuesto o a la sanción disciplinaria.

- Inexistencia de un sistema de información, que incluya base de datos esenciales que posibilite realizar procesos de evaluación.

- No se ha generado en el nivel institucional un "espacio reconocido" en el tiempo de trabajo académico para encarar esta actividad.

- Inexistencia del financiamiento necesario para generar un proceso de evaluación y asegurar su permanencia en el tiempo de modo ininterrumpido.

- Inexistencia de un reconocimiento y estimulación acordes con las exigencias que se derivan de una opción de dedicación exclusiva y un compromiso ligado a lo institucional.

- Desconocimiento de la comunidad universitaria respecto a los modelos y metodologías propuestas desde los niveles de dirección para los procesos de evaluación universitaria.

\section{Procedimiento operacional de la acreditación institucional}

A continuación se presentan los principales momentos que conlleva la realización del proceso de acreditación institucional:

- Primera aproximación al proyecto o programa objeto de evaluación (Estudio documental).

- Constitución y capacitación del equipo central coordinador de todo el proceso. 
- Constitución y capacitación del equipo técnico que participará en cada uno de los diferentes procesos y áreas evaluativos.

- Construcción de los instrumentos para la recolección de datos de cada proceso evaluativo por áreas.

- Validación de los instrumentos con responsables del proyecto o programa.

- Aplicación de los instrumentos a todos los actores involucrados y previstos.

- Análisis e interpretación de los resultados por los responsables.

- Metaevaluación: evaluación de la autoevaluación (Conclusiones).

- Confección o evaluación del proyecto de desarrollo institucional.

- Designación o contratación de evaluadores externos.

- Realización de la evaluación externa nacional o internacional.

- Confrontación de los resultados de la evaluación externa con los de la autoevaluación para hacer las conclusiones.

- Realizar la certificación académica o no.

\section{Etapas de desarrollo de la autoevaluación}

Resulta conveniente estructurar el proceso de autoevaluación por etapas, del modo siguiente. 11

\section{1a Etapa: Propósitos y capacitación}

Consiste en establecer claramente el propósito de la autoevaluación, recopilación de antecedentes teóricos y prácticos, elaboración del material que se utilizará en seminarios y de preferencia hacer participar en cursos o talleres de formación y capacitación a los evaluadores que formarán parte de la comisión de evaluación (grupo coordinador y grupos ejecutores o evaluadores).

Es importante la difusión del proceso, así como despertar el interés y la motivación de toda la comunidad que participará en el proceso.

\section{2a Etapa: Programa de actividades}

Elaborar un programa de actividades detallado, con la finalidad de preparar y diseñar todo el proceso de recolección y análisis de la información, lo que debe ir acompañado de una agenda de trabajo que considere los recursos humanos, materiales y económicos necesarios.

Es importante considerar:

- La conformación de los grupos de trabajo.

- El apoyo administrativo necesario.

- La ubicación de los locales de trabajo.

- La definición de tiempos y periodos.

- La recopilación de información, tanto de hechos como de opiniones de los distintos factores e instancias internas y externas del sistema en estudio.

Ejemplo de hechos:

- Datos del personal docente (perfiles académicos, funciones, responsabilidades, producción y otros) y estudiantes (caracterización socioeconómica, académica y de origen, índices académicos de regularidad y otros).

- Plan de estudios (mapa curricular, distribución de cursos, actividades extracurriculares, proceso de enseñanza y aprendizaje y otros).

- Recursos (fuentes de financiamiento, infraestructura, equipos, bibliografía y otros).

Como fuentes de opinión se utilizan:

\begin{tabular}{|l|rl|}
\hline - Personal docente. & - Servicios de apoyo. \\
- Estudiantes. & - Agencias externas. \\
- Graduados. & - Instituciones similares. \\
- Ejecutivos. & - Pares académicos. \\
\hline
\end{tabular}

Se contempla la aplicación y procesamiento de encuestas, realización de entrevistas, la revisión de datos y verificación de hechos y cifras, construcción de índices y juicios de opinión, elaboración de informes técnicos de distintas funciones, realización de reuniones de la comisión, discusiones y conclusiones.

La etapa tiene como propósito detectar, caracterizar y proponer soluciones sobre las fortalezas, oportunidades, debilidades y amenazas de los distintos elementos internos y externos del quehacer académico, los cuales se identifican con las categorías y estándares de la acreditación.

El informe de autoevaluación responde a un diagnóstico de la realidad de la carrera y deberá exponer un plan de trabajo, cuyas condiciones deben ser validadas por los evaluadores externos. 


\section{3a Etapa: Plan de acción}

Una vez emitido el informe de los evaluadores, se consolida en el informe una propuesta de mejoramiento y desarrollo que debe ser negociada y acordada como plan de implantación derivado de la autoevaluación y sus consecuencias.

Lo anterior debe derivar en un plan de acción común con la administración central de la institución (plan de desarrollo institucional), en el que se comprometan recursos, personal, infraestructura, equipos y capacidades a favor de resultados de superación en las áreas con problemas.

\section{4a Etapa: Seguimiento de la implementación del plan de acción}

Es necesario establecer una fase de monitoreo y seguimiento de la implementación del plan de acción. Esta etapa es dinámica en tanto permite introducir las rectificaciones que necesite el propio plan. Los resultados de las mediciones deben cotejarse con los valores designados en los estándares, ya sean propios de la carrera o externos.

Finalmente se recomienda como metodología de utilidad para el proceso de autoevaluación, la siguiente:

- Definición del objeto de estudio.

- Definición de los sujetos que participarán en el diagnóstico (promotores, equipo técnico y población consultada).

- Definición de estrategias, selección del conjunto de acciones que aseguran la detección de problemas y la participación de los sujetos (curso, taller, aplicación de instrumentos, recuperación de la información, seguimiento de graduados).

- Establecimiento de criterios, principios que servirán de referencia para la emisión de juicios de valor para definir las conclusiones de cada problema detectado y proponer alternativas de solución a mediano y largo plazo.

- Análisis preliminar del objeto. A la luz de los criterios para detectar discrepancias.

- Establecimiento de hipótesis de trabajo. Ordenar las actividades de diagnósticos basadas en su importancia o urgencia.

- Selección de la información más relevante sobre el objeto de estudio, los criterios e hipótesis de registros administrativos, fuentes personales, fuentes de grupo y otras.

- Organización de actividades de participación. Analizar, seleccionar y estructurar los mecanismos y acciones que deberán realizarse sistemática y ordenadamente para que los miembros de la comunidad tengan la posibilidad de intervenir activamente en la detección y análisis de los problemas.

- Recolección de la información en todas las actividades para sistematizarla.

- Integración del diagnóstico, consistente en sistematizar y analizar la información obtenida a la luz de los criterios, la proposición de alternativas de solución especificadas en objetivos, acciones concretas y señalamiento de plazos de cumplimiento, así como el próximo plazo de reevaluación.

- Sometimiento del documento o informe final a la consideración de toda la comunidad de la institución, así como el plan de desarrollo institucional resultante.

- Establecimiento de los procedimientos y plazos para el monitoreo y control del plan de desarrollo institucional.

\section{MÉTODO}

Diseño experimental, recolección de la información, técnicas a ser utilizadas, tamaño de la muestra poblacional, pasos o etapas del estudio, estrategia de investigación, análisis estadístico.

El ámbito espacial será la Facultad de Ciencias Administrativas de la UNMSM y el ámbito temporal será el año 2012.

El universo de estudio estará conformado por la Facultad de Ciencias Administrativas de la UNMSM con sus (03) tres escuelas académico profesionales de: Administración, Administración de Turismo y Administración de Negocios Internacionales, que al año 2011 se encuentran registradas formalmente. Se incluiran a todos los docentes nombrados, asimismo a todos los profesores contratados y trabajadores administrativos con mas de (02) dos años de servicios.

La unidad de análisis estará conformada por las autoridades, docentes, personal administrativo y estudiantes de la Facultad de Ciencias Administrativas de la UNMSM. 
La muestra de investigación estará constituida por el $50 \%$ de los actores involucrados en el quehacer universitario, especialmente las autoridades, docentes, personal administrativo y estudiantes de las tres (03) escuelas académico profesionales de la Facultad de Ciencias Administrativas de la UNMSM.

En relacion con los instrumentos se aplicarán, los cuestionarios y entrevistas para recopilar información relativa a la autoevaluación de la Facultad de ciencias Administrativas de la UNMSM, el cuestionario será aplicado al personal docente, personal administrativo y alumnos de las tres escuelas académico profesional que conforman la Facultad, asimismo se aplicarán entrevistas a las autoridades, otro instrumento sera el análisis documental a través de la la revisión de la Ley Universitaria, el estatuto, reglamentos y normas internas de la universidad.

\section{RESULTADOS}

\section{Factor Currículo}

Tabla $\mathrm{N}^{\circ} 1$

\begin{tabular}{|c|c|c|c|c|c|}
\hline \multirow{2}{*}{ Pregunta $N^{\circ} 1$} & \multicolumn{5}{|c|}{ RESPUESTA TOTAL } \\
\hline & SI & $\%$ & NO & $\%$ & $\%$ \\
\hline $\begin{array}{l}\text { ¿El actual currículo contiene la fundamentación, el perfil profesional, el plan de } \\
\text { estudios, las sumillas, la malla curricular, los recursos humanos y físicos? }\end{array}$ & 9 & 45 & 11 & 55 & 100 \\
\hline $\begin{array}{l}\text { Análisis e Interpretación: } \\
\text { El } 45 \% \text { de los encuestados respondió positivamente, mientras que el } 55 \% \text { re- } \\
\text { spondió negativamente, por lo que se concluye que el actual currículo no cuenta } \\
\text { con los requisitos que demanda la acreditación. }\end{array}$ & & & & & \\
\hline
\end{tabular}

Tabla $\mathrm{N}^{\circ} 2$

\begin{tabular}{|l|c|c|c|c|c|}
\hline \multirow{2}{*}{ Pregunta $\mathbf{N}^{\circ} \mathbf{2}$} & \multicolumn{4}{|c|}{ RESPUESTA TOTAL } \\
\cline { 2 - 6 } & SI & $\%$ & NO & $\%$ & $\%$ \\
\hline ¿La carrera o especialidad responde a la demanda social actual y futura? & 14 & 70 & 6 & 30 & 100 \\
\hline $\begin{array}{l}\text { Análisis e Interpretación: } \\
\text { El } 70 \% \text { de los encuestados opinó afirmativamente, mientras que el 30\% lo hizo } \\
\text { negativamente, por lo que se concluye que la carrera 0 especialidad responde a } \\
\text { la demanda actual y futura. }\end{array}$ & & & & & \\
\hline
\end{tabular}

Tabla $\mathrm{N}^{\circ} 3$

\begin{tabular}{|c|c|c|c|c|c|}
\hline \multirow{2}{*}{ Pregunta $\mathrm{N}^{\circ} 3$} & \multicolumn{5}{|c|}{ RESPUESTA TOTAL } \\
\hline & SI & $\%$ & NO & $\%$ & $\%$ \\
\hline $\begin{array}{l}\text { ¿El perfil del egresado está definido de forma clara, precisa y es de conocimiento } \\
\text { público? }\end{array}$ & 7 & 35 & 13 & 65 & 100 \\
\hline $\begin{array}{l}\text { Análisis e Interpretación: } \\
\text { Se observa que el } 35 \% \text { de los encuestados respondió afirmativamente, en tanto el } \\
65 \% \text { lo hizo negativamente, por lo que se puede deducir que el perfil del egresado } \\
\text { no está definido en forma clara. }\end{array}$ & & & & & \\
\hline
\end{tabular}

Tabla $\mathrm{N}^{\circ} 4$

\begin{tabular}{|c|c|c|c|c|c|}
\hline \multirow{2}{*}{ Pregunta $\mathrm{N}^{\circ} 4$} & \multicolumn{5}{|c|}{ RESPUESTA TOTAL } \\
\hline & SI & $\%$ & NO & $\%$ & $\%$ \\
\hline ¿Existe coherencia entre el perfil del egresado y las exigencias profesionales? & 5 & 25 & 15 & 75 & 100 \\
\hline $\begin{array}{l}\text { Análisis e Interpretación: } \\
\text { El } 25 \% \text { opinó positivamente, mientras el } 75 \% \text { negativamente, porque se con- } \\
\text { cluye que no existe coherencia entre el perfil del egresado y las exigencias } \\
\text { profesionales. }\end{array}$ & & & & & \\
\hline
\end{tabular}


Tabla $N^{\circ} 5$

\begin{tabular}{|c|c|c|c|c|c|}
\hline \multirow{2}{*}{ Pregunta $\mathrm{N}^{\circ} 5$} & \multicolumn{5}{|c|}{ RESPUESTA TOTAL } \\
\hline & SI & $\%$ & NO & $\%$ & $\%$ \\
\hline $\begin{array}{l}\text { ¿El perfil del egresado define los conocimientos, las capacidades, las habilidades, } \\
\text { las actitudes y competencias a adquirirse? }\end{array}$ & 10 & 50 & 10 & 50 & 100 \\
\hline $\begin{array}{l}\text { Análisis e Interpretación: } \\
\text { En la tabla se observa que el } 50 \% \text { de los encuestados opina positivamente, en } \\
\text { tanto el otro } 50 \% \text { lo hizo negativamente, por lo que se concluye que el perfil del } \\
\text { egresado no estaría definiendo adecuadamente los conocimientos, habilidades, } \\
\text { actitudes y competencias. }\end{array}$ & & & & & \\
\hline
\end{tabular}

\section{Factor Enseñanza - Aprendizaje}

Tabla $N^{\circ} 6$

\begin{tabular}{|c|c|c|c|c|c|}
\hline \multirow{2}{*}{ Pregunta $\mathrm{N}^{\circ} 1$} & \multicolumn{5}{|c|}{ RESPUESTA TOTAL } \\
\hline & SI & $\%$ & NO & $\%$ & $\%$ \\
\hline $\begin{array}{l}\text { ¿Se utilizan métodos, técnicas y sistemas para el proceso enseñanza - apren- } \\
\text { dizaje }\end{array}$ & 15 & 75 & 5 & 25 & 100 \\
\hline $\begin{array}{l}\text { Análisis e Interpretación: } \\
\text { El } 75 \% \text { opina favorablemente, mientras el } 25 \% \text { opina desfavorablemente, porque } \\
\text { se deduce la utilización de métodos, técnicas y sistemas en el proceso enseñan- } \\
\text { za-aprendizaje. }\end{array}$ & & & & & \\
\hline
\end{tabular}

Tabla $\mathrm{N}^{\circ} 7$

\begin{tabular}{|c|c|c|c|c|c|}
\hline \multirow{2}{*}{ Pregunta $\mathrm{N}^{\circ} 2$} & \multicolumn{5}{|c|}{ RESPUESTA TOTAL } \\
\hline & SI & $\%$ & NO & $\%$ & $\%$ \\
\hline $\begin{array}{l}\text { ¿Existe coordinación entre las áreas académicas y administrativas que intervienen } \\
\text { en el proceso enseñanza aprendizaje? }\end{array}$ & 8 & 40 & 12 & 60 & 100 \\
\hline $\begin{array}{l}\text { Análisis e Interpretación: } \\
\text { El } 40 \% \text { de los encuestados respondió afirmativamente, mientras que el } 60 \% \\
\text { negativamente, por lo que podemos concluir que no existe coordinación entre las } \\
\text { tareas académicas y administrativas que intervienen en el proceso enseñanza- } \\
\text { aprendizaje. }\end{array}$ & & & & & \\
\hline
\end{tabular}

Tabla $\mathrm{N}^{\circ} 8$

\begin{tabular}{|c|c|c|c|c|c|}
\hline \multirow{2}{*}{ Pregunta $\mathrm{N}^{\circ} 3$} & \multicolumn{5}{|c|}{ RESPUESTA TOTAL } \\
\hline & SI & $\%$ & NO & $\%$ & $\%$ \\
\hline $\begin{array}{l}\text { ¿Existe coherencia entre los objetivos, los métodos y los instrumentos de } \\
\text { evaluación? }\end{array}$ & 6 & 30 & 14 & 70 & 100 \\
\hline $\begin{array}{l}\text { Análisis e Interpretación: } \\
\text { El } 70 \% \text { de los encuestados opinó positivamente y el } 30 \% \text { lo hizo negativamente, } \\
\text { por lo que se deduce que si existe coherencia entre los objetivos, los métodos y } \\
\text { los instrumentos de evaluación. }\end{array}$ & & & & & \\
\hline
\end{tabular}

Tabla $\mathrm{N}^{\circ} 9$

\begin{tabular}{|c|c|c|c|c|c|}
\hline \multirow{2}{*}{ Pregunta $\mathrm{N}^{\circ} 4$} & \multicolumn{5}{|c|}{ RESPUESTA TOTAL } \\
\hline & SI & $\%$ & NO & $\%$ & $\%$ \\
\hline $\begin{array}{l}\text { ¿Existen actividades orientadas a desarrollar capacidades específicas del } \\
\text { perfil del egresado? }\end{array}$ & 9 & 45 & 11 & 55 & 100 \\
\hline $\begin{array}{l}\text { Análisis e Interpretación: } \\
\text { Mientras el } 45 \% \text { opina afirmativamente, el } 55 \% \text { lo hace negativamente, } \\
\text { por lo que se concluye que no existen actividades orientadas a desarrollar } \\
\text { capacidades específicas del perfil del egresado. }\end{array}$ & & & & & \\
\hline
\end{tabular}


Tabla $\mathrm{N}^{\circ} 10$

\begin{tabular}{|l|c|c|c|c|c|}
\hline \multirow{2}{*}{ Pregunta $\mathbf{N}^{\circ} \mathbf{5}$} & \multicolumn{4}{|c|}{ RESPUESTA TOTAL } \\
\cline { 2 - 5 } & SI & $\%$ & NO & $\%$ & $\%$ \\
\hline $\begin{array}{l}\text { ¿Existen modalidades de obtención del grado académico de bachiller y del título } \\
\text { profesional? }\end{array}$ & 20 & 100 & 00 & 00 & 100 \\
\hline $\begin{array}{l}\text { Análisis e Interpretación: } \\
\text { En relación con esta pregunta, el 100\% respondió que existen modalidades de } \\
\text { obtención del grado académico de bachiller y título profesional. }\end{array}$ & & & & \\
\hline
\end{tabular}

\section{Factor Investigación}

Tabla $\mathrm{N}^{\circ} 11$

\begin{tabular}{|l|c|c|c|c|c|}
\hline \multirow{2}{*}{ Pregunta No 1} & \multicolumn{4}{|c|}{ RESPUESTA TOTAL } \\
\cline { 2 - 6 } & SI & $\%$ & NO & $\%$ & $\%$ \\
\hline ¿Existe apoyo para la publicación de trabajos de investigación? & 11 & 55 & 9 & 45 & 100 \\
\hline $\begin{array}{l}\text { Análisis e Interpretación: } \\
\text { El } 55 \% \text { de los encuestados opinó afirmativamente, mientras que el 45\% lo hizo } \\
\text { negativamente, por lo que se deduce que sí existe apoyo para la publicación de } \\
\text { trabajos de investigación. }\end{array}$ & & & & & \\
\hline
\end{tabular}

Tabla $\mathrm{N}^{\circ} 12$

\begin{tabular}{|l|c|c|c|c|c|}
\hline \multirow{2}{*}{ Pregunta N² } & \multicolumn{4}{|c|}{ RESPUESTA TOTAL } \\
\cline { 2 - 5 } & SI & $\%$ & NO & $\%$ & $\%$ \\
\hline $\begin{array}{l}\text { ¿Existe un procedimiento para la actualización curricular que incorpore los } \\
\text { resultados de la investigación realizada? }\end{array}$ & 10 & 50 & 10 & 50 & 100 \\
\hline $\begin{array}{l}\text { Análisis e Interpretación: } \\
\text { El } 50 \% \text { opina positivamente, mientras que el otro 50\% negativamente, por lo que } \\
\text { podemos concluir que sí existe un procedimiento para la actualización curricular } \\
\text { que incorpore los resultados de la investigación realizada. }\end{array}$ & & & & \\
\hline
\end{tabular}

Tabla $\mathrm{N}^{\circ} 13$

\begin{tabular}{|c|c|c|c|c|c|}
\hline \multirow{2}{*}{ Pregunta $\mathrm{N}^{\circ} 3$} & \multicolumn{5}{|c|}{ RESPUESTA TOTAL } \\
\hline & SI & $\%$ & NO & $\%$ & $\%$ \\
\hline $\begin{array}{l}\text { ¿Existen espacios de discusión entre los docentes, alumnos y la comunidad } \\
\text { acerca de las investigaciones relacionadas con la carrera? }\end{array}$ & 5 & 25 & 15 & 75 & 100 \\
\hline $\begin{array}{l}\text { Análisis e Interpretación: } \\
\text { En la tabla se observa que solo el } 25 \% \text { opinó positivamente, en tanto el } 75 \% \text { opinó } \\
\text { negativamente, por lo que se concluye que no existen espacios de discusión entre } \\
\text { los docentes, alumnos y la comunidad acerca de las investigaciones relacionadas } \\
\text { con la carrera. }\end{array}$ & & & & & \\
\hline
\end{tabular}

Tabla $\mathrm{N}^{\circ} 14$

\begin{tabular}{|c|c|c|c|c|c|}
\hline \multirow{2}{*}{ Pregunta $\mathrm{N}^{\circ} 4$} & \multicolumn{5}{|c|}{ RESPUESTA TOTAL } \\
\hline & SI & $\%$ & NO & $\%$ & $\%$ \\
\hline ¿Existen seguimientos del avance de las investigaciones? & 15 & 75 & 5 & 25 & 100 \\
\hline $\begin{array}{l}\text { Análisis e Interpretación: } \\
\text { En la tabla se observa que el } 75 \% \text { de los encuestados opinó favorablemente, } \\
\text { mientras el } 25 \% \text { lo hizo negativamente, por lo que se pude deducir que sí existen } \\
\text { seguimientos del avance de las investigaciones. }\end{array}$ & & & & & \\
\hline
\end{tabular}


Tabla $\mathrm{N}^{\circ} 15$

\begin{tabular}{|l|c|c|c|c|c|}
\hline \multirow{2}{*}{ Pregunta $\mathbf{N}^{\circ} \mathbf{5}$} & \multicolumn{4}{|c|}{ RESPUESTA TOTAL } \\
\cline { 2 - 5 } & SI & $\%$ & NO & $\%$ & $\%$ \\
\hline ¿Existen seguimientos de la inversión de los recursos de investigación? & 11 & 55 & 9 & 45 & 100 \\
\hline $\begin{array}{l}\text { Análisis e Interpretación: } \\
\text { El } 55 \% \text { de los encuestados respondió afirmativamente, mientras el 45\% lo hizo } \\
\text { negativamente, por lo que se concluye que sí existe seguimiento de la inversión } \\
\text { de los recursos de investigación. }\end{array}$ & & & & & \\
\hline
\end{tabular}

Tabla $\mathrm{N}^{\circ} 16$

\begin{tabular}{|c|c|c|c|c|c|}
\hline \multirow{2}{*}{ Pregunta $N^{\circ} 6$} & \multicolumn{5}{|c|}{ RESPUESTA TOTAL } \\
\hline & SI & $\%$ & NO & $\%$ & $\%$ \\
\hline $\begin{array}{l}\text { ¿Participan los docentes a dedicación exclusiva y tiempo completo en actividades } \\
\text { de investigación? }\end{array}$ & 10 & 50 & 10 & 50 & 100 \\
\hline $\begin{array}{l}\text { Análisis e Interpretación: } \\
\text { En esta pregunta hubo un equilibrio o empate en las respuestas, por lo que } \\
\text { podemos deducir que los docentes a dedicación exclusiva y tiempo completo par- } \\
\text { ticipan en actividades de investigación. }\end{array}$ & & & & & \\
\hline
\end{tabular}

Tabla $\mathrm{N}^{\circ} 17$

\begin{tabular}{|c|c|c|c|c|c|}
\hline \multirow{2}{*}{ Pregunta $\mathrm{N}^{\circ} 7$} & \multicolumn{5}{|c|}{ RESPUESTA TOTAL } \\
\hline & SI & $\%$ & NO & $\%$ & $\%$ \\
\hline $\begin{array}{l}\text { ¿Existe un sistema de selección y participación del alumno en los proyectos de } \\
\text { investigación y desarrollo tecnológico? }\end{array}$ & 5 & 25 & 15 & 75 & 100 \\
\hline $\begin{array}{l}\text { Análisis e Interpretación: } \\
\text { En la tabla se observa que solo el } 25 \% \text { opina favorablemente, en tanto el } 75 \% \\
\text { opina desfavorablemente, por lo que se concluye que no existe un sistema de } \\
\text { selección y participación del alumno en los proyectos de investigación y desarrollo } \\
\text { tecnológico. }\end{array}$ & & & & & \\
\hline
\end{tabular}

Tabla $\mathrm{N}^{\circ} 18$

\begin{tabular}{|c|c|c|c|c|c|}
\hline \multirow{2}{*}{ Pregunta $\mathrm{N}^{\circ} 8$} & \multicolumn{5}{|c|}{ RESPUESTA TOTAL } \\
\hline & SI & $\%$ & NO & $\%$ & $\%$ \\
\hline $\begin{array}{l}\text { ¿Existe un sistema de obtención, administración y control de la financiación para } \\
\text { programas de investigación y desarrollo tecnológico? }\end{array}$ & 8 & 40 & 12 & 60 & 100 \\
\hline $\begin{array}{l}\text { Análisis e Interpretación: } \\
\text { Solo el } 40 \% \text { de los encuestados opina positivamente, frente al } 60 \% \text { que opina } \\
\text { desfavorablemente, por lo que se concluye que no existe un sistema de obten- } \\
\text { ción, administración y control de la financiación para programas de investigación } \\
\text { y desarrollo tecnológico. }\end{array}$ & & & & & \\
\hline
\end{tabular}

Tabla $\mathrm{N}^{\circ} 19$

\begin{tabular}{|c|c|c|c|c|c|}
\hline \multirow{2}{*}{ Pregunta $\mathrm{N}^{\circ} 9$} & \multicolumn{5}{|c|}{ RESPUESTA TOTAL } \\
\hline & SI & $\%$ & NO & $\%$ & $\%$ \\
\hline $\begin{array}{l}\text { ¿Existen normas que regulan la distribución de los beneficios intelectuales } 0 \\
\text { materiales que puedan surgir de la investigación? }\end{array}$ & 11 & 55 & 9 & 45 & 100 \\
\hline $\begin{array}{l}\text { Análisis e Interpretación: } \\
\text { El } 55 \% \text { de los encuestados opina positivamente, mientras el } 45 \% \text {, negativamente, } \\
\text { por lo que se deduce que sí existen normas que regulan la distribución de los } \\
\text { beneficios intelectuales y materiales que puedan surgir de la investigación. }\end{array}$ & & & & & \\
\hline
\end{tabular}


Tabla $\mathrm{N}^{\circ} 20$

\begin{tabular}{|c|c|c|c|c|c|}
\hline \multirow{2}{*}{ Pregunta $\mathrm{N}^{\circ} 10$} & \multicolumn{5}{|c|}{ RESPUESTA TOTAL } \\
\hline & SI & $\%$ & NO & $\%$ & $\%$ \\
\hline $\begin{array}{l}\text { ¿Existen publicaciones de los últimos } 5 \text { años y los medios adecuados para su } \\
\text { divulgación? }\end{array}$ & 15 & 75 & 5 & 25 & 100 \\
\hline $\begin{array}{l}\text { Análisis e Interpretación: } \\
\text { El } 75 \% \text { de los encuestados respondió afirmativamente, frente al } 25 \% \text { que lo hizo } \\
\text { negativamente, por lo que se concluye que sí existen publicaciones de los últimos } \\
5 \text { años y los medios adecuados para su divulgación. }\end{array}$ & & & & & \\
\hline
\end{tabular}

Tabla $\mathrm{N}^{\circ} 21$

\begin{tabular}{|c|c|c|c|c|c|}
\hline \multirow{2}{*}{ Pregunta $\mathrm{N}^{\circ} 11$} & \multicolumn{5}{|c|}{ RESPUESTA TOTAL } \\
\hline & SI & $\%$ & NO & $\%$ & $\%$ \\
\hline $\begin{array}{l}\text { ¿La investigación desarrollada contribuye con la innovación y el avance científico } \\
\text { y tecnológico? }\end{array}$ & 15 & 75 & 5 & 25 & 100 \\
\hline $\begin{array}{l}\text { Análisis e Interpretación: } \\
\text { El } 75 \% \text { de los encuestados opina favorablemente, mientras que el } 25 \% \text { lo hizo } \\
\text { negativamente, por lo que se deduce que la investigación desarrollada contribuye } \\
\text { con la innovación y el avance científico y tecnológico. }\end{array}$ & & & & & \\
\hline
\end{tabular}

\section{Factor Extensión Universitaria y Proyección Social}

Tabla $\mathrm{N}^{\circ} 22$

\begin{tabular}{|c|c|c|c|c|c|}
\hline \multirow{2}{*}{ Pregunta $\mathrm{N}^{\circ} 1$} & \multicolumn{5}{|c|}{ RESPUESTA TOTAL } \\
\hline & SI & $\%$ & NO & $\%$ & $\%$ \\
\hline $\begin{array}{l}\text { ¿Se realizan actividades que divulguen la cultura hacia la sociedad, propiciando la } \\
\text { creatividad, la transformación tecnológica, la promoción de talentos y el desarrollo } \\
\text { de valores? }\end{array}$ & 12 & 60 & 8 & 40 & 100 \\
\hline $\begin{array}{l}\text { Análisis e Interpretación: } \\
\text { En la tabla se observa que el } 60 \% \text { de los encuestados responde afirmativamente, } \\
\text { frente al } 40 \% \text { que lo hace negativamente, por lo que se deduce que sí se realizan } \\
\text { actividades que divulgan la cultura hacia la sociedad propiciando la creatividad, la } \\
\text { transformación tecnológica, la promoción de talentos y el desarrollo de valores. }\end{array}$ & & & & & \\
\hline
\end{tabular}

Tabla $\mathrm{N}^{\circ} 23$

\begin{tabular}{|l|c|c|c|c|c|}
\hline \multirow{2}{*}{ Pregunta $\mathbf{N}^{\circ} 2$} & \multicolumn{5}{|c|}{ RESPUESTA TOTAL } \\
\cline { 2 - 6 } & SI & $\%$ & NO & $\%$ & $\%$ \\
\hline ¿Existen cursos de actualización profesional y de capacitación? & 16 & 80 & 4 & 20 & 100 \\
\hline $\begin{array}{l}\text { Análisis e Interpretación: } \\
\text { Ante la pregunta, se observa que el 80\% responde afirmativamente, mientras el } \\
20 \% \text { lo hace negativamente, por lo que se concluye que sí existen cursos de actu- } \\
\text { alización profesional y de capacitación. }\end{array}$ & & & & & \\
\hline
\end{tabular}

Tabla $\mathrm{N}^{\circ} 24$

\begin{tabular}{|c|c|c|c|c|c|}
\hline \multirow{2}{*}{ Pregunta $\mathrm{N}^{\circ} 3$} & \multicolumn{5}{|c|}{ RESPUESTA TOTAL } \\
\hline & SI & $\%$ & NO & $\%$ & $\%$ \\
\hline $\begin{array}{l}\text { ¿Existe vinculación con el sector productivo que permita el intercambio de } \\
\text { conocimientos? }\end{array}$ & 9 & 45 & 11 & 55 & 100 \\
\hline $\begin{array}{l}\text { Análisis e Interpretación: } \\
\text { El } 45 \% \text { de los encuestados responde afirmativamente, mientras el } 55 \% \text { lo hace } \\
\text { negativamente, por lo que se concluye que no existe vinculación con el sector } \\
\text { productivo que permita el intercambio de conocimientos. }\end{array}$ & & & & & \\
\hline
\end{tabular}


Tabla $\mathrm{N}^{\circ} 25$

\begin{tabular}{|c|c|c|c|c|c|}
\hline \multirow{2}{*}{ Pregunta $\mathrm{N}^{\circ} 4$} & \multicolumn{5}{|c|}{ RESPUESTA TOTAL } \\
\hline & SI & $\%$ & NO & $\%$ & $\%$ \\
\hline $\begin{array}{l}\text { ¿Existen programas que promuevan la realización de eventos científicos y } \\
\text { tecnológicos }\end{array}$ & 8 & 40 & 12 & 60 & 100 \\
\hline $\begin{array}{l}\text { Análisis e Interpretación: } \\
\text { En la tabla se observa que solo el } 40 \% \text { opina favorablemente, frente a un } 60 \% \text { que } \\
\text { opina desfavorablemente, por lo que se concluye que no existen programas que } \\
\text { promuevan la realización de eventos científicos y tecnológicos. }\end{array}$ & & & & & \\
\hline
\end{tabular}

Tabla $\mathrm{N}^{\circ} 26$

\begin{tabular}{|l|l|l|l|l|l|}
\hline \multirow{2}{*}{ Pregunta $\mathbf{N}^{\circ} \mathbf{5}$} & \multicolumn{5}{|c|}{ RESPUESTA TOTAL } \\
\cline { 2 - 6 } & SI & $\%$ & NO & $\%$ & $\%$ \\
\hline $\begin{array}{l}\text { ¿Existe un sistema administrativo eficiente y transparente en la gestión de la } \\
\text { prestación de servicios? }\end{array}$ & 0 & 00 & 20 & 100 & 100 \\
\hline $\begin{array}{l}\text { Análisis e Interpretación: } \\
\text { En la tabla se observa que el 100\% de los encuestados respondió negativamente, } \\
\text { por lo que se deduce que no existe un sistema administrativo eficiente y transpar- } \\
\text { ente en la gestión de la prestación de servicios. }\end{array}$ & & & & & \\
\hline
\end{tabular}

Tabla $\mathrm{N}^{\circ} 27$

\begin{tabular}{|c|c|c|c|c|c|}
\hline \multirow{2}{*}{ Pregunta $N^{\circ} 6$} & \multicolumn{5}{|c|}{ RESPUESTA TOTAL } \\
\hline & SI & $\%$ & NO & $\%$ & $\%$ \\
\hline $\begin{array}{l}\text { ¿Existe un sistema de producción de bienes y prestación de servicios a terceros, } \\
\text { que involucra al personal docente, administrativo y alumnos? }\end{array}$ & 0 & 00 & 20 & 100 & 100 \\
\hline $\begin{array}{l}\text { Análisis e Interpretación: } \\
\text { El } 100 \% \text { de los encuestados opinó negativamente, por lo que se concluye que no } \\
\text { existe un sistema de producción de bienes y servicios a terceros que involucre al } \\
\text { personal docente, administrativo y alumnos. }\end{array}$ & & & & & \\
\hline
\end{tabular}

Nota: Se aplicaron 20 encuestas a docentes de la Facultad de Ciencias Administrativas de la UNMSM.

\section{REFERENCIAS BIBLIOGRÁFICAS}

Rodríguez A. (1996) "Calidad de la Educación Superior. El sistema de evaluación y acreditación en la Universidad Mayor de San Simón de Cochabamba, Bolivia”. En: Conferencia Regional para la transformación de la Educación Superior en América Latina y el Caribe. La Habana: CRESALC/MES; 1996.

Arríen JB. (1996) "Calidad y acreditación: exigencias a la Universidad". En: Conferencia Regional sobre Políticas y Estrategias para la Transformación de la Educación Superior en América Latina y el Caribe. La Habana: CRESALC/MES; 1996.

Villarroel C. (1996) “Calidad y acreditación universitarias latinoamericanas para Latinoamérica”. En: Conferencia Regional sobre Políti- cas y Estrategias para la Transformación de la Educación Superior en América Latina y el Caribe. La Habana: CRESALC/MES; 1996.

Aljure E, Hakim R. (1996) "Lineamientos para la acreditación de la Educación Superior en Colombia”. En: Conferencia Regional sobre Políticas y Estrategias para la Transformación de la Educación Superior en América Latina y el Caribe. La Habana: CRESALC/ MES; 1996.

Trindade H.(1996) "Evaluación institucional y calidad académica: resistencia y construcción". En: Conferencia Regional sobre Políticas y Estrategias para la Transformación de la Educación Superior en América Latina y el Caribe... La Habana: CRESALC/MES; 1996. 
México. Centro de Asesoría Pedagógica de la Universidad Anáhuac. Evaluación institucional y calidad. Experiencia en Educación Básica de la Universidad de Anáhuac. $<$ http://mec.es/cide/rieme/documentos/ lopez/lopez.pdf. Diciembre 2002>.

Cañete Escalona F. (1998) "La Evaluación Institucional: ¿Qué tiene la Escuela? ¿Qué entrega la Escuela? Enfoques Educativos" 1998; $<$ http://rehue,csociales.uchile.cl/publicaciones/enfoques/01/.Diciembre 2002>.

España. Consejo de Universidades. Plan Nacional de Evaluación Calidad Universitaria. Evaluación institucional.
$<$ http://www.uma.es/ccalidad/evaluacióninstitucional.htm. Diciembre 2002.>

Simoneau R. (2002) “La Evaluación Institucional: conceptos teóricos. Academia de Evaluación Institucional”. Documentos de trabajo. México 2002.

$<$ http://valle-mexico.pm.org/evins/. Diciembre 2002.>

Argentina. Manual de Acreditación para carreras de Ingeniería en la República Argentina. $<$ http://www.fceia.unr.edu.or/.../acreditacion-archivo_pdf/pa_Acreditacion_confedi_Manual-pdf. Diciembre 2002>. 